\title{
POSITIONAL ACCURACY ASSESSMENT USING SINGLE AND MULTI-GNSS
}

\author{
H. H. Garcia ${ }^{1}$, M. E. Mercurio ${ }^{2}$, D. P. Noveloso ${ }^{1}$, R. B. Reyes ${ }^{1, *}$ \\ ${ }^{1}$ Department of Geodetic Engineering, College of Engineering, University of the Philippines Diliman - \\ hannagarcia@yahoo.com,dpnoveloso@up.edu.ph,rbreyes3@up.edu.ph \\ ${ }^{2}$ Department of Geodetic Engineering, College of Engineering, Visayas State University - mrtnmercurio22@ gmail.com
}

\section{Commission IV}

KEY WORDS: Single GNSS, Multi-GNSS, Circular Error Probable (CEP), 2 Dimensional RMS (2DRMS), RTKLIB, Positioning

\begin{abstract}
:
Global Navigation Satellite System (GNSS) provides users worldwide with a three-dimensional positioning, velocity and time solution 24-hour service using transmitted radio signals from orbiting satellites in space. Dependency on the number of satellites available during observation can improve satellite geometry and increase redundancy which be a factor for the quality of GNSS positioning result. Each position derived from different combinations of satellite systems were evaluated aside from using GPS only. The test is conducted by single and relative positioning method using survey grade receivers. Post-processing of GNSS data were processed in an open source software, RTKLIB, getting the RMS and 2DRMS of each position derived from different combination. The resulting satellite system combination for single positioning for horizontal were better considering the inclusion of GPS and BeiDou. However, position accuracy degrades when the inclusion of GLONASS and/or GALILEO during the processing. For relative positioning, position derived from the inclusion of GALILEO, BeiDou and QZSS degrades the results. For height results in single positioning method, the displacement from the mean value is about 4.00 meters which is derived from Galileo only satellite system.
\end{abstract}

\section{INTRODUCTION}

Global Navigation Satellite System (GNSS) is the collective term for those navigation systems that provide users with a threedimensional positioning solution by passive ranging using radio signals transmitted by orbiting satellites (Groves, 2008). It is an all-weather, operating 24-hour, and global in-service coverage (Toluc, 2016). For the United States is the Global Positioning System (GPS), the European Union is the GALILEO, for Russians is the GLONASS, for the People's Republic of China is the BeiDou, and for the Japanese is the QZSS. GPS and GLONASS are both qualified for global coverage applications (Nur, Song, Sung, \& Chung, 2016). Furthermore, GALILEO and BeiDou were expected to operate in full global coverage by 2020 aside from its localization coverage (Perez-Ruiz \& Upadyaya, 2012). However, QZSS satellite orbit is in geosynchronous to ensure one satellite is over Japan and covering the Asia-Pacific region (Maeda, 2005). With numerous available satellites in space, the multi-constellation of GNSS provides better satellite geometry, increases satellite redundancy, resulting to accurate position determination and time convergence (Rabbou \& ElRabbany, 2017). For the GNSS application, the position determination is the concerned activity then the navigation and timing (Sickle, 2016).

Signal quality and number of available satellites are factors for the accurateness of the station position using GNSS receivers (Gülmez \& Tuşat, 2017). Single point and static positioning method of data collection are implemented using different combinations of satellite systems such as GPS, GLONASS, BeiDou, QZSS, and GALILEO. Single point method is characterized by using a single receiver which requires a minimum of four (4) satellites available during measurement. For static positioning mode, the receiver is motionless throughout the observation (Sickle, 2016). The latter method employs relative positioning which uses an active geodetic base station and another (static) station that observes same satellite simultaneously (Ragheb \& Ragab, 2012).
Describing the quality of GNSS positioning is by accuracy and precision in which accuracy refers to the truth while precision refers to how close to the mean observation. The GNSS positioning accuracy can be expressed as a percent of the data within a distance from the averaged location, and more common terms used are Circular Error Probable (CEP), Root Mean Square error (RMS), and Distance Root Mean Square error (DRMS). The CEP is the radius of circle containing $50 \%$ of measured horizontal positions by GNSS are within the zone while the 2DRMS refers to $95 \%$ of the observed positions were within the distance of DRMS multiplied twice (Nur et al., 2016). Both measures are for horizontal position only.

As mentioned above, results were mainly affected by different errors that were inherited throughout the GNSS observation. These errors include the satellite position error, clock error on receivers, ionospheric and atmospheric delays, and multipath (Min, Ehsani, \& Salyani, 2008). Additionally, the geometry and number of available satellites observed by the receivers can also influence to the quality of the position results (Nur et al., 2016).

The study aims to determine the average position of station using a survey grade receiver through different combinations of satellite systems by single and relative positioning mode. Results are compared between satellite system combinations for single and relative positioning. The study specifically aims to (i) postprocess GNSS data using RTKLIB application, (ii) derive position results from different satellite system combination on different modes, and (iii) analyse and compare the quality of positions derived.

\section{DATA AND METHODS}

The general method for the activity is composed of three components such as the data gathering, data processing, and data analysis. The following flow procedure is shown in Figure 1 


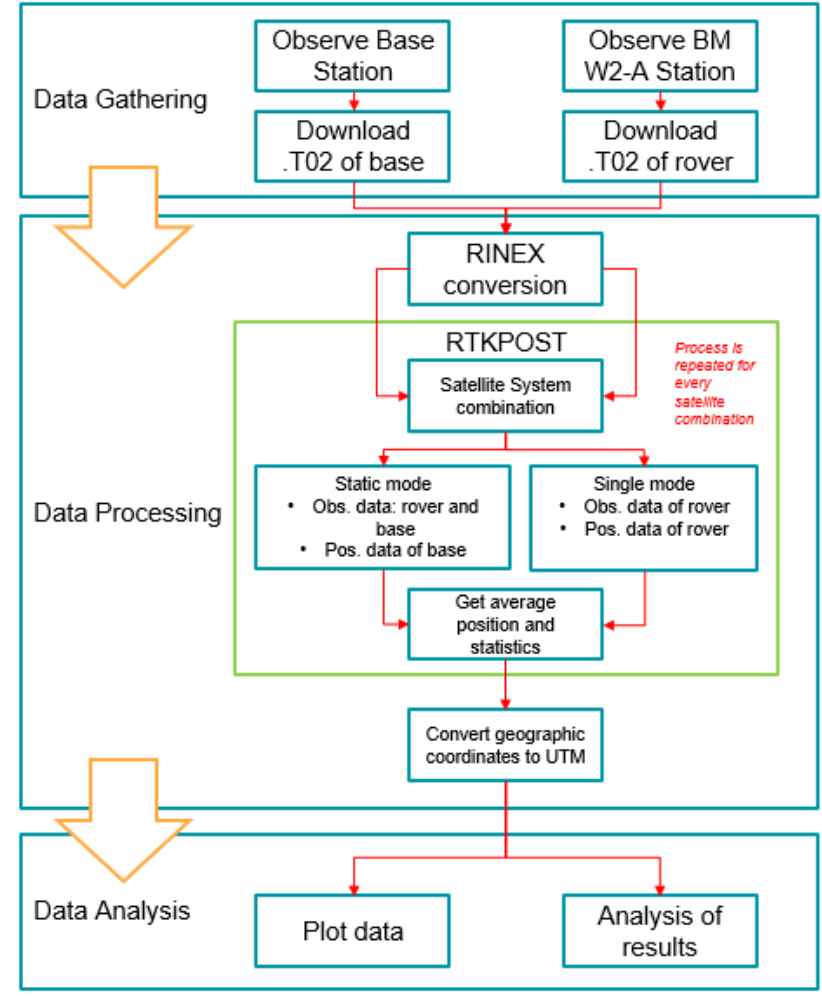

Figure 1. Process of the activity

\subsection{Study Area}

Two stations were used in this study, one is a ground monumented station and the other is an active geodetic station. The ground station (BM W2-A), which is an elevation benchmark, was located along University Avenue entrance leading to the University of the Philippines, Diliman, Quezon City. The active geodetic station was located at the rooftop of Melchor Hall, College of Engineering. This station, which is approximately 1.5 kilometers away from BM W2-A, will be used as a base station in the relative positioning mode.

\subsection{Data Gathering}

The observed station is in stationary mounted on the tripod using Trimble SPS985 GNSS receiver or a span of one (1) hour from $1430 \mathrm{H}$ to $1530 \mathrm{H}$ on March 16, 2019. A smartphone was used to control the GNSS receiver through Wi-Fi connection and downloaded the .T02 data from the receiver for post-process.

\subsection{Processing}

The .T02 data files were converted into RINEX format to produce the observation and position files. These converted files were the input for processing in RTKPOST to derive the station position. Each satellite positions were derived from difference and multiple satellite combinations e.g. GPS only, GPS \& GALILEO, GPS-BeiDou-GLONASS, etc

Two processing method were implemented in RTKPOST, first is for single positioning method and the second is the relative (static mode) positioning determination. Average results were recorded for each combination and their respective statistics. The derived geographic coordinates were converted to UTM (zone 51N) using a converter in spreadsheet since the statistics results are in meters. The process is repeated until all satellite combinations were processed.

\subsection{Analysis}

There are two types of statistical accuracy used in this study, twice the distance root mean square or 2DRMS (Eq. 1), and the circular error probability or CEP (Eq. 2). The positions were then plotted compared to the accuracy based from the positions derived from the influence of different satellite system combination.

$$
\begin{aligned}
& 2 D R M S=2 \times \sqrt{\left(\sigma_{x}^{2}+\sigma_{y}^{2}\right)} \\
& C E P=0.59\left(\sigma_{x}+\sigma_{y}\right)
\end{aligned}
$$

where $\sigma_{x}=$ standard deviation of easting value $\sigma_{\mathrm{y}}=$ standard deviation of northing value

\section{RESULTS}

As shown in Figure 2, there were 34 to 40 satellites vehicles (SV) were observed, consisting satellites from GPS (G), GLONASS (R), GALILEO (E), BeiDou (B), and QZSS (Q). Majority of the observed SVs is in north-south orientation and only few in eastwest orientation.

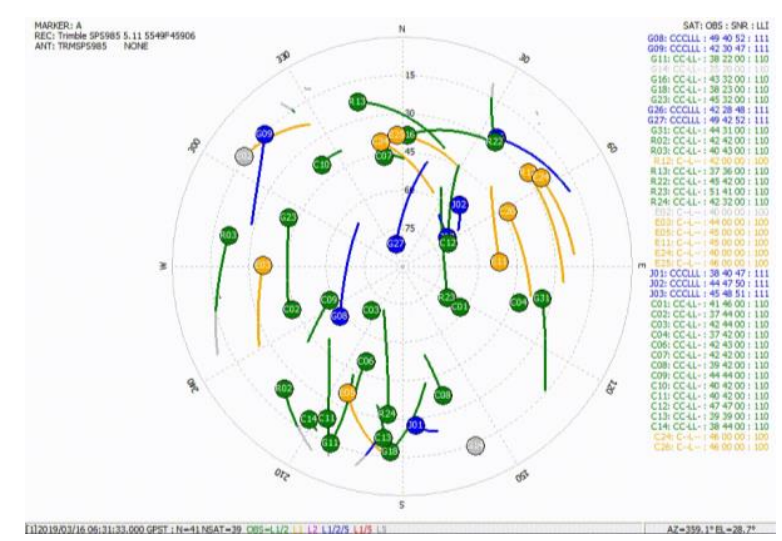

Figure 2. Skyplot of observed satellite vehicles

\subsection{Single Positioning}

Large RMS values were computed to the satellite system combinations mentioned above especially for the Up component of the positions determined that reaches 4 meters (Figure 3). For North and East component results from other combinations were consistently low as expected from the previous results determined before.

Figure 4 shows the test accuracies of each results in which satellite systems from R and RE fails to meet the 2DRMS test. A significant result pointed out to $\mathrm{R}$ satellite influence on other satellite system such as E and Q combinations.

\subsection{Static Positioning}

For static positioning, Figure 5 shows the RMS results from the combinations; a low value of RMS was computed except for E and QE satellites which its Up-component deviates about $40 \mathrm{~cm}$. Other components of the positions from different systems shows better results about less than $10 \mathrm{~cm}$ in RMS values. 
RMS of Single Positioning
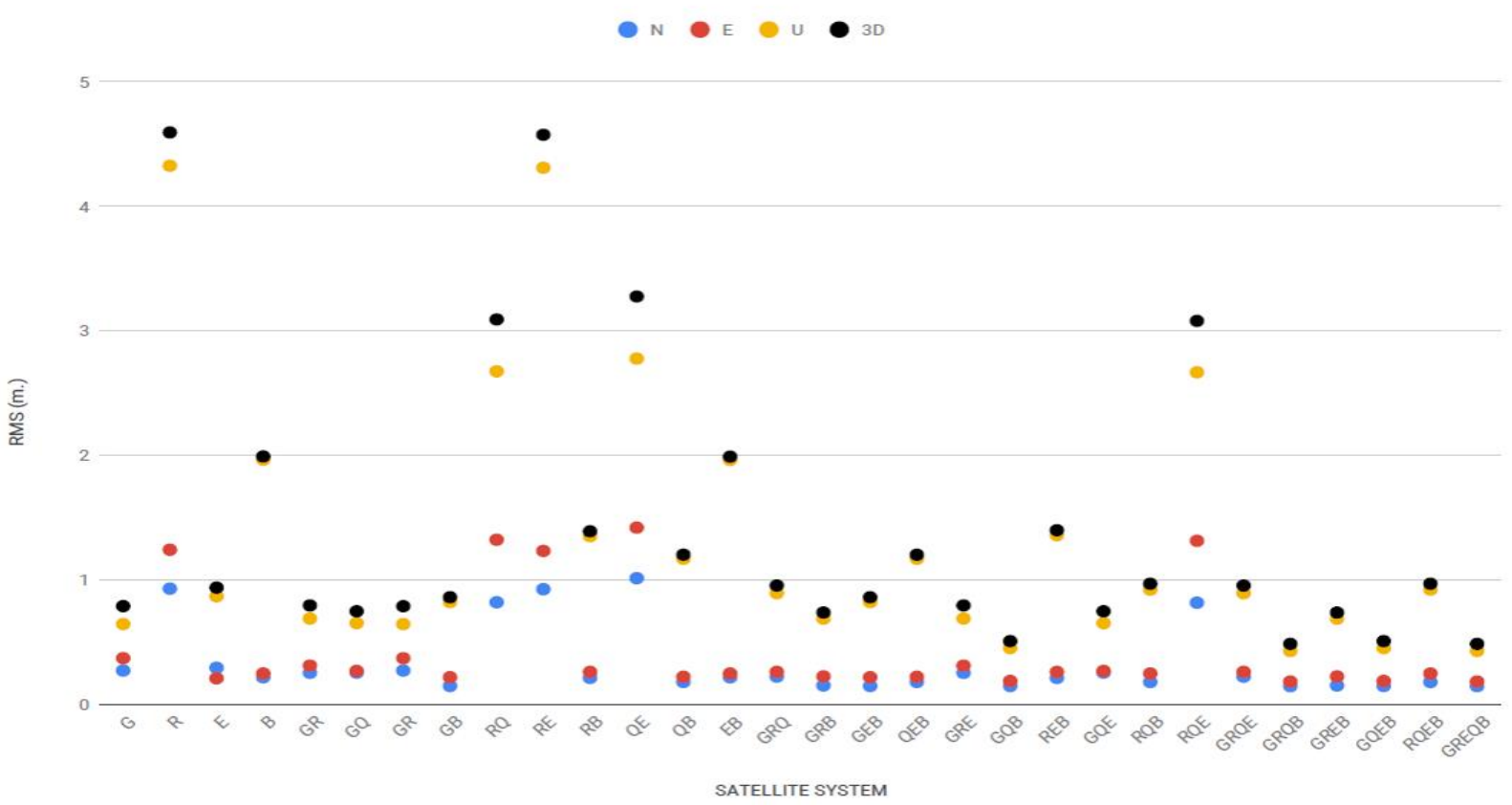

Figure 3. RMS of single positioning

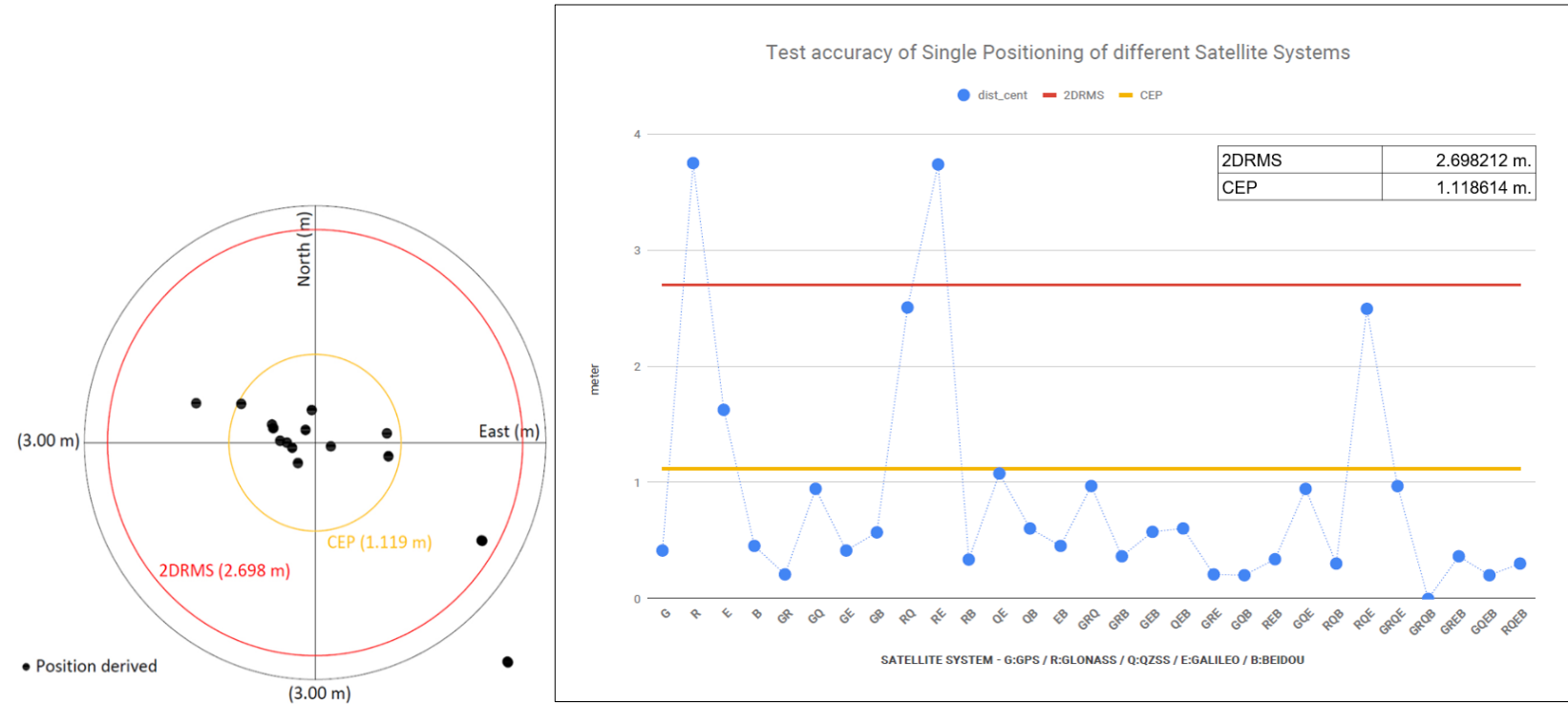

Figure 4. Test accuracy of single positioning 


\section{RMS of Static Positioning}

0.4

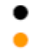

0.3
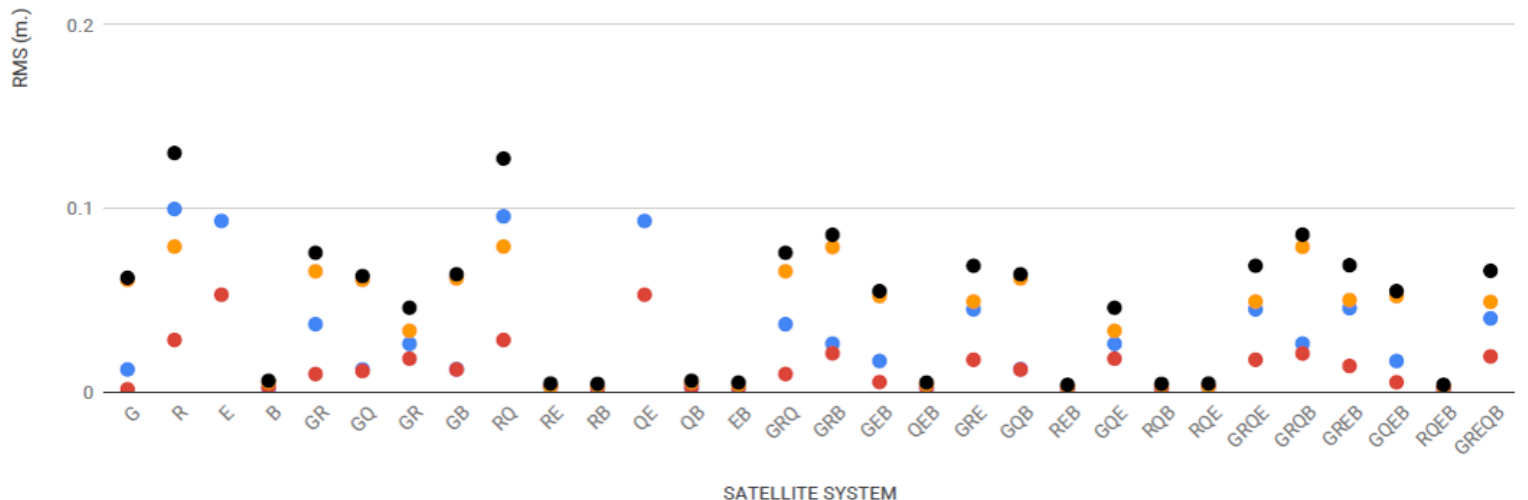

Figure 5. RMS of static positioning

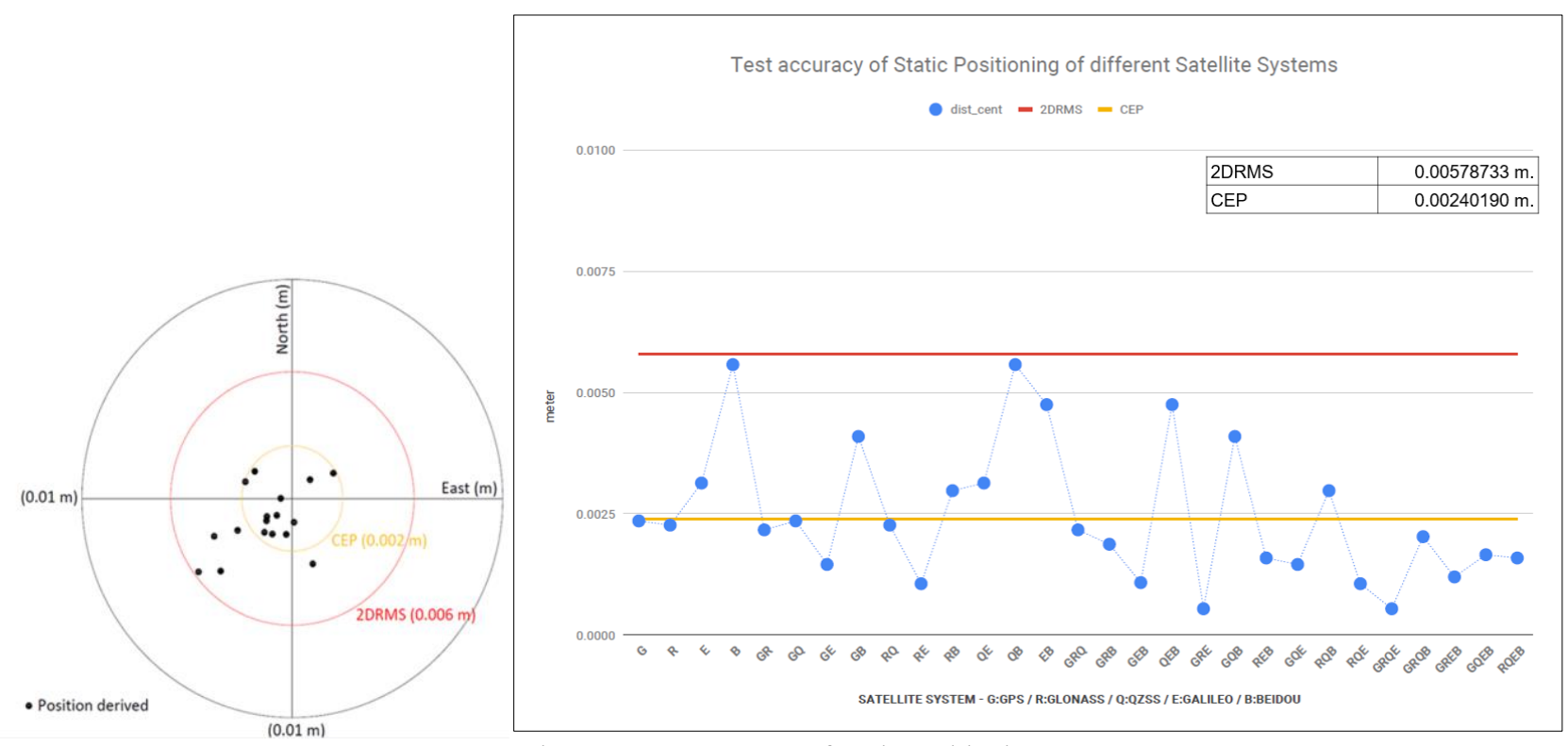

Figure 6. Test accuracy of static positioning 


\section{CONCLUSION}

In single satellite system, positions derived from $\mathrm{G}$ and $\mathrm{B}$ passed the CEPS while $\mathrm{R}$ and $\mathrm{E}$ failed. The $\mathrm{E}$ solution is within the threshold of 2DRMS but R exceeded said criteria. For dual GNSS combinations, positions derived from GR, GQ, GE, GB, RB, QE, $\mathrm{QB}$, and $\mathrm{EB}$ are within the CEP value while RQ and RE were beyond. However, RQ barely passed the 2DRMS. For multiple GNSS combinations, 14 out of 15 combinations passed the CEP and 2DRMS values. In relative positioning, all single, dual and multi-combination GNSS passed the 2DRMS value. Additionally, the height results showed that for single positioning the $\mathrm{E}$ derived position differ largely from the mean by about 4 meters. Based on the results, horizontal positioning is more accurate when $\mathrm{G}$ satellites were included. It was observed that the inclusion of $\mathrm{R}$ satellites for single positioning degraded the positional accuracy with high displacement of about $3.7 \mathrm{~m}$. Furthermore, in relative positioning, contributions of $\mathrm{E}$ and $\mathrm{B}$ satellites degrade the quality of the results when paired with $\mathrm{Q}$ and R satellites.

\section{REFERENCES}

Groves, P. D. (2008). Principles of GNSS, Inertial, and Multisensor Integrated Navigation Systems.

Gülmez, S., \& Tuşat, E. (2017). The Analysis of GPS Data in Different Observation Periods Using Online GNSS Process Services Since: 2009. International Journal of Environment and Geoinformatics, 4(1).

Maeda, H. (2005). QZSS overview and interoperability. In 18th International Tech. Meeting of the Satellite Division of the Institute of Navigation. Long Beach.

Min, M., Ehsani, R., \& Salyani, M. (2008). Dynamic accuracy of GPS receivers in citrus orchard. Appl. Eng. Agric., 24(6), 861868 .

Nur, S., Song, M., Sung, N., \& Chung, S. (2016). Performance Comparison of Single and Multi-GNSS Receivers under Agricultural Fields in Korea Engineering in Agriculture, Environment and Food Performance comparison of single and multi-GNSS receivers under agricultural fields in Korea. Engineering in Agriculture, Environment and Food, 9(1), 27-35. https://doi.org/10.1016/j.eaef.2015.09.002

Perez-Ruiz, M., \& Upadyaya, S. K. (2012). GNSS in precision agricultural operations. In: New Approach of Indoor and Outdoor Localization Systems. Tech Europe, 3-26.

Rabbou, M. A., \& El-Rabbany, A. (2017). Performance analysis of precise point positioning using multi-constellation GNSS: GPS, GLONASS, Galileo and BeiDou. Survey Review, (May 2016). https://doi.org/10.1080/00396265.2015.1108068

Ragheb, A. E., \& Ragab, A. F. (2012). Enhancement of GPS Single Point Positioning Accuracy Using Referenced Network Stations, 18(10),1463-1474.

https://doi.org/10.5829/idosi.wasj.2012.18.10.2752

Sickle, J. Van. (2016). GPS for Land Surveyors, 4th Edition. CRC Press (Vol. 82). https://doi.org/10.14358/pers.82.3.181
Toluc, A. B. (2016). Multi-GNSS Precise Point Positioning Using GPS, GLONASS and Galileo THESIS. Ohio State University. 